Patients may present acutely with bowel obstruction due to the tumour itself or the effects of desmoplasia. Desmoplasia can occur and may progress even after medical treatments. Patients with bowel ischaemia maybe managed with anticoagulation. It is difficult to differentiate desmoplasia from adhesions in obstructive patients. The median survival in the symptomatic group was 15 months less than the total median OS, this may reflect the complex nature of patients' disease status.

These patients have complex symptomatology which can only be dealt with by experienced multidisciplinary approach. Further research is required into the pathogenesis of desmoplasia to enable better treatment.

Competing interests None.

Keywords desmoplasia, midgut, neuroendocrine tumour (NET).

\section{PTU-100 MORBIDITY \& MORTALITY ASSOCIATED WITH DESMOPLASIA IN MIDGUT (CARCINOID) NEUROENDOCRINE TUMOURS}

doi:10.1136/gut.2011.239301.228

F El-Khouly,* M S Khan, C Toumpanakis, G Ogunbiyi, M Winslet, M Caplin Neuroendocrine Tumour Unit, Royal Free Hampstead NHS Trust, London, $U K$

Introduction Midgut neuroendocrine tumours (NETs) form part of a heterogenous group characterised by their ability to secrete hormones and other mediators which may cause carcinoid syndrome. They may also present with complex problems related to fibrosis/desmoplasia. Products including 5-HT secreted by NETs may induce fibrosis. It has been previously reported that $30 \%$ of patients are found to have desmoplasia on CT at presentation while the incidence increases as disease progresses. Desmoplasia may cause symptoms secondary to mesenteric vein occlusion, ischaemia as well as bowel obstruction. Aim To assess morbidity and mortality associated with desmoplasia in midgut NETs.

Methods A retrospective review of our NET Unit database for patients with a diagnosis of midgut NET and desmoplasia. Information available included: age, sex, diagnosis, previous treatment, current management and outcomes from MDT meetings. 107 midgut NET patients with desmoplasia were identified. Patients with non-midgut NETs were excluded. Overall survival (OS) was calculated from time of diagnosis.

Results Full data was available for 99 patients. 51 male and 48 female patients. Desmoplasia was identified at surgery or at CT imaging. 61 (62\%) patients were symptomatic from either obstructive or ischaemic cause at some point. 68 (69\%) patients had undergone surgical procedures, including primary resection, re-resection, stoma formation and bypass. Of the 48 patients who had undergone surgical resection of the primary tumour, 31 (65\%) had presented with obstructive symptoms. 38 (38\%) were asymptomatic and desmoplasia was diagnosed incidentally on CT. $6(6 \%)$ patients were symptomatic from SMV occlusion needing anticoagulation. $5(5 \%)$ patients were treated with long-term TPN. 2 had short bowel syndrome. 36 patients had died. Total overall median survival was 96 months. Overall median survival in the symptomatic group was 81 months and not reached in the asymptomatic group $(\log$-rank $\mathrm{p}=0.059)$.

Conclusion Over half of patients with midgut NETs with desmoplasia are symptomatic needing surgical intervention. 